\title{
Altered Oncogene Activity Contributes to Compensation for Antisense Suppression of Bcl-2 and Tumor Resistance
}

\author{
Marvin Rubenstein 1,2,3,4*, Courtney M. P. Hollowell2, Patrick Guinan"1,2,45 \\ ${ }^{1}$ Division of Cellular Biology, Hektoen Institute for Medical Research, Chicago, IL, USA \\ ${ }^{2}$ Division of Urology, Stroger Hospital of Cook County, Chicago, IL, USA \\ ${ }^{3}$ Department of Biochemistry, Rush University Medical Center, Chicago, IL, USA \\ ${ }^{4}$ Department of Urology, Rush University Medical Center, Chicago, IL, USA \\ ${ }^{5}$ Department of Urology, University of Illinois at Chicago, Chicago, IL, IL, USA \\ Email: DrMarv@Prodigy.net
}

Received 28 May 2015; accepted 19 July 2015; published 22 July 2015

Copyright (C) 2015 by authors and Scientific Research Publishing Inc.

This work is licensed under the Creative Commons Attribution International License (CC BY). http://creativecommons.org/licenses/by/4.0/

(c) (i) Open Access

\section{Abstract}

Antisense oligonucleotides (oligos) have targeted growth regulatory proteins in prostate cancer models. To identify compensatory alterations in the expression of non-targeted genes we evaluate mono- and bispecific oligos targeting and equally suppressing the expression of the apoptosis inhibitory protein bcl-2. Bcl-2 is chosen because oligos directed towards it have entered clinical trials to restore apoptosis in cancer patients. Treated LNCaP cells compensate for the diminished bcl-2 by suppressing caspase-3 (an apoptosis promoter) while enhancing expression of AKT-1 (another apoptosis inhibitor), androgen receptor (AR) and its (p300 and IL-6) coactivators. Additional proteins are enhanced including PD-1, its ligand PD-L1 (immune checkpoint blockade markers) and fas-ligand, which activate apoptosis through the signal transduction, along with suppressor protein p53, polymerase transcription mediator MED-12 and signal transducer STAT-3. These alterations in expression may contribute to a greatly enhanced expression of the proliferation marker KI-67. This suggests that therapeutic approaches to restore apoptosis through suppression of bcl-2 lead to an altered expression in non-targeted genes involving apoptosis, androgen sensitivity, transcriptional activity and immune responsiveness, leads to an increase in proliferation (and a more androgen driven aggressive phenotype). In this study we evaluate the expression of two oncogenes ( $\mathrm{v}$-myc and $\mathrm{K}$-ras) and find a large and significant enhancement of v-myc activity, which is produced by oligos targeting bcl-2 at the $5^{\prime}$ position. For K-ras, although significant suppression is produced by the bispecific targeting bcl-2 at the 3' position, the percent change is relatively small compared with other compensatory alterations we have measured, and much less than in v-myc. Therefore, for the two oncogenes being evaluated,

${ }^{*}$ Corresponding author.

How to cite this paper: Rubenstein, M., Hollowell, C.M.P. and Guinan, P. (2015) Altered Oncogene Activity Contributes to Compensation for Antisense Suppression of Bcl-2 and Tumor Resistance. Open Journal of Apoptosis, 4, 62-70.

http://dx.doi.org/10.4236/ojapo.2015.43007 
only increased v-myc activity is probably large enough to contribute to increased tumor aggressiveness in compensation for bcl-2 suppression.

\author{
Keywords
}

Antisense Oligonucleotides, Prostate Cancer, Bcl-2, Gene Compensation, Therapy

\title{
1. Introduction
}

Gene therapy is in theory specific, but encounters difficulties in practice. While suitable targets are found in many pathways, and tumors express altered patterns of gene expression, the actual activity of most regulatory genes is similar to normal cells. Resistance develops because the biochemical pathways being involved are complex and regulated by combinations of both stimulatory and inhibitory factors; many affected by therapy and some of these affected genes are susceptible to altered expressions having the potential to reverse the effects of the intended treatment (such as the clinical reestablishment or enhancement of apoptosis). Our studies have shown that in the LNCaP prostate cancer model, following what is assumed to be specific suppression of bcl-2 by antisense oligonucleotides (oligos), tumors may resist these attempts by altering their dependence on a variety of non-targeted gene products to replace the activity of the altered process (apoptosis) and develop a more aggressive, androgen driven, phenotype through the process of compensation [1]. Just as bacteria and viruses mutate to evade antibiotic and antiviral agents, tumor cells are under similar selective pressure to evade therapy. Whether is chemically induced (chemotherapy) or not, that based on suppression of gene translation (including oligo mediated gene therapy). Although oligo based therapy has already reached clinical trials to treat a variety of tumors, the unintended compensatory consequences of intervention are poorly understood, and can contribute to the emergence and selection of more aggressive cells. Specifically, antisense oligos have been administered against inhibitors of apoptosis (bcl-2 and clusterin) together with radio [2] [3] or chemotherapy [4] in attempting to restore that process mediated by the mitochondrial pathway for the treatment of human prostate tumors. Additional targets to regulate apoptosis can also include those involved with activation mediated by signal transduction and initiated via cell surface receptors and their respective ligands (PD-1/PD-L1 and fas/fas-ligand) [5]. If such therapy is to be successful, it is important to examine all mechanisms by which tumors compensate and become resistant, particularly where (as in apoptosis) there may be several pathways towards activation.

We previously reported that LNCaP cells treated with antisense oligos directed against bcl-2 (administered in a nanoparticle suspension of lipofectin) compensate by suppressing caspase-3 (1) (an apoptosis promoter) and enhanced AKT-1(an inhibitor of apoptosis) [6], androgen receptor [7] (AR), co-activating p300 [8] and IL-6 expression [9]. Of clinical importance, for AR, p300 and IL-6, their new patterns of expression are often associated with more aggressive tumors [9]. In addition, PD-1, its ligand PD-L1 (immune checkpoint blockade markers) and fas-ligand, which activate apoptosis through signal transduction, are also enhanced [5] (presumably leading to further inhibition of T-cell activity), as are suppressor protein p53 [10], polymerase transcription mediator MED-12 [11], signal transducer STAT-3 [6] and KI-67 (manuscript submitted) a marker are associated with increased tumor proliferation (and aggression), which may be the ultimate result of these altered gene expressions. Monoclonal antibody therapy directed towards PD-1/PD-L1 is attracting increased attention for treatment of solid tumors (particularly melanoma, but also prostate) since PD-L1 has expression on many cell types including $\mathrm{T}$, tumor, epithelial and endothelial cells [12]. This pathway also activates apoptosis via signal transduction, so compensatory changes are not unexpected following bcl-2 suppression.

From our studies we conclude that, in LNCaP, therapeutic approaches to restore apoptosis (including the use of antisense oligos) which suppress bcl-2 can lead to altered expression in non-targeted genes and regulatory changes not only involving apoptosis, but also androgen sensitivity, transcriptional activity and immune responsiveness. Many of androgen related alterations are similar to expression patterns associated with more advanced prostate tumors, while regulation of apoptosis involved both mitochondrial and signal transducing pathways.

We now evaluate the expression of oncogenes v-myc and K-ras. Avian myelocytomatosis viral oncogene homolog (v-myc) is a nuclear phosphoprotein involved in the cell cycle, apoptosis and transformation, acting as a transcription factor [13]. Kirsten ras oncogene homolog (K-ras) is a member of the GTPase family. When mutated it induces several types of malignancy [14]. 
For gene therapy to ultimately be successful it must be made more specific and mechanisms of compensation must be identified and possibly targeted for additional suppression. This could include the use of mono-specific gene regulatory (oligos) agents employed simultaneously, but could also include either bispecific or even (proposed) multispecific oligo based agents [15], directed towards multiple genes affecting growth, apoptosis (death) or proliferation (KI-67 and cyclins). We have suggested that such agents could include oligos intended to suppress both KI-67 and bcl-2 (manuscript under review); perhaps additional oligos should target oncogenes as well.

\section{Methods}

\subsection{Oligonucleotides}

Oligos (mono- or bispecific) were purchased from Eurofins MWG Operon (Huntsville, AL). Each was phosphorothioated on three terminal bases at 5' and 3' positions. Stock solutions were made to a final concentration of $625 \mu \mathrm{M}$ in sterile Dulbecco PBS.

\section{Base Sequences}

Each oligo contained at least one CAT sequence and targeted the area adjacent to the mRNA AUG initiation codon for the respective targeted protein (EGFR or bcl-2).

$\mathbf{M R}_{4}$ (monospecific targeting bcl-2) T-C-T-C-C-C-A-G-C-G-T-G-C-G-C-C-A-T

$\mathbf{M R}_{24}$ (bispecific targeting EGFR/bcl-2) G-A-G-G-G-T-C-G-C-A-T-C-G-C-T-G-C-T-C-T-C-T-C-C-C-A-GC-G-T-G-C-G-C-C-A-T

$\mathbf{M R}_{42}$ (bispecific targeting bcl-2/EGFR) T-C-T-C-C-C-A-G-C-G-T-G-C-G-C-C-A-T-G-A-G-G-G-T-C-G-CA-T-C-G-C-T-G-C-T-C

\subsection{Cell Culture}

LNCaP cells (American Type Culture Collection [ATCC]) were grown in RPMI 1640 supplemented with 10\% bovine serum, $1 \%$ L-glutamine and $1 \%$ penicillin/streptomycin in a $5 \% \mathrm{CO}_{2}$ incubator. Log phase cells were harvested using EDTA/trypsin and equally distributed into $75 \mathrm{~cm}^{2}$ flasks (Corning, NY). At intervals media were either supplemented or replaced with fresh.

\subsection{Oligo Treatment Prior to PCR}

When cell density in the culture flasks approached $75 \%$ confluence (4 days prior to oligo addition), $10 \mathrm{ml}$ of additional fresh media was added. Cells were incubated for another 3 days before $5 \mathrm{ml}$ of media was replaced with fresh the day before oligos were added. $100 \mu \mathrm{l}$ of stock oligos were added (in RPMI 1640 media) to bring the final concentration to $6.25 \mu \mathrm{M}$. Incubation proceeded for an additional 24 hours in the presence or absence of monospecific $\mathrm{MR}_{4}$, or the $\mathrm{MR}_{24}$ and $\mathrm{MR}_{42}$ bispecifics.

\subsection{RNA Extraction}

Following treatment, media was removed, a single $\mathrm{ml}$ of cold $\left(4^{\circ} \mathrm{C}\right)$ RNAzol B was added to each $75 \mathrm{~cm}^{2}$ culture flask and the monolayer lysed by repeated passage through a pipette. All procedures were performed at $4^{\circ} \mathrm{C}$. The lysate was removed, placed in a centrifuge tube to which $0.2 \mathrm{ml}$ of chloroform was added, and shaken. The mixture stayed on ice for $5 \mathrm{~min}$, was spun at 12,000 g for $15 \mathrm{~min}$, and the upper aqueous volume removed and placed in a fresh tube. An equal volume of isopropanol was added, the tube shaken, and allowed to stay at $4^{\circ} \mathrm{C}$ for 15 min before similar centrifugation to pellet the RNA. The supernatant was removed, the pellet washed in a single ml of $75 \%$ ethanol, then spun for $8 \mathrm{~min}$ at $7500 \mathrm{~g}$. The ethanol was pipetted off and the formed pellet air dried at $-20^{\circ} \mathrm{C}$.

\subsection{RNA Quantitation}

RNA was resuspended in $250 \mu \mathrm{l}$ of DEPC treated $\mathrm{H}_{2} \mathrm{O}$, and quantitated using a Qubit florometer and Quant-iT RNA assay kit (Invitrogen). DEPC is an inhibitor of RNase activity.

\subsection{RT-PCR}

Extracted RNA was diluted in DEPC treated water to $40 \mu \mathrm{g} / \mu \mathrm{l} .1$ - $4 \mu \mathrm{l}$ of this RNA was added to $1 \mu \mathrm{l}$ of both 
sense and antisense primers (forward and reverse sequences) for actin, bcl-2, v-myc and K-ras. From a kit purchased from Invitrogen the following reactants were added for RT-PCR: $25 \mu \mathrm{l}$ of $2 \times$ reaction mixture, $2 \mu \mathrm{l} \mathrm{Su}-$ perScript III RT/platinum Taq mix, tracking dye, and $3 \mu \mathrm{l} \mathrm{MgSO}_{4}$ (of a $5 \mathrm{mM}$ stock concentration). DEPC treated water was added to yield a final volume of $50 \mu$ l. RT-PCR was performed for $2 \times 25$ cycles using the F54 program in a Sprint PCR Thermocycler. As a control for RT-PCR product production, human actin expression was tested in RNA extracted from HeLa cells which was provided in a kit purchased from Invitrogen (in the reaction mixture, no $\mathrm{MgSO}_{4}$ was included, the difference compensated for by $3 \mu \mathrm{l}$ of DEPC treated water).

\section{Primers}

Primer sequences were obtained from the National Center Biotechnology Information (NCBI) website: http://www.ncbi.nlm.nih.gov/nuccore/NM_031966

They were designed to amplify $100-300 \mathrm{bp}$ fragments and anneal between $58-60{ }^{\circ} \mathrm{C}$.

\section{1) Actin}

Forward primer sequence: 5' CAA ACA TGA TCT GGG TCA TCT TCT C 3'

Reverse primer sequence: 5' GCT CGT CGT CGA CAA CGG CTC

PCR product produced was 353 bp in length

\section{2) Bcl-2}

Forward primer sequence: 5' GAG ACA GCC AGG AGA AAT CA 3'

Reverse primer sequence: 5' CCT GTG GAT GAC TGA GTA CC 3'

PCR product produced was $127 \mathrm{bp}$ in length.

3) v-myc

Forward primer sequence: 5' CGA CGA GAC CTT CAT CAA AA 3'

Reverse primer sequence: 5' TGC TGT CGT TGA GAG GGT AG 3'

PCR product produced was 248 bp in length.

\section{4) K-ras}

Forward primer sequence: 5' TGC ATT TTT CCT TTG AGT GC 3'

Reverse primer sequence: 5' CAC ACT GCA TAT GTC CCA CA 3'

PCR product produced was $153 \mathrm{bp}$ in length.

\subsection{Detection and Quantitation of Product}

\subsubsection{Agarose Gel Electrophoresis}

$1.5 \%$ agarose gels were prepared in a $50 \mathrm{ml}$ volume of TBE buffer $(1 \times$ solution: $0.089 \mathrm{M}$ Tris borate and 0.002 M EDTA, pH 8.3), containing $3 \mu \mathrm{l}$ of ethidium bromide in a Fisher Biotest electrophoresis system. Samples were run for 2 hours at a constant voltage of 70 using a BioRad 1000/500 power supply source. To locate the amplified PCR product, $3 \mu \mathrm{l}$ of a molecular marker (Invitrogen) which contained a sequence of bases in 100 base pair increments (Invitrogen) as well as $2 \mu \mathrm{l}$ of a sucrose based bromphenol blue tracking dye were run in each gel.

\subsubsection{Quantitation}

Gels were visualized under UV light and photographed using a Canon ELPH 300 HS PowerShot digital camera. Photos were converted to black and white format and bands quantitated using Mipav software provided by the National Institute of Health. Means and standard deviations were compared using Student $t$-tests to determine significance.

\section{Results}

\subsection{Bcl-2 Expression}

As a control for RT-PCR product production, human actin expression was tested in RNA extracted from HeLa cells. Figure 1 provides an example of one such band (actin) suitable for scanning and quantitation. The molecular weight markers shown in the left column are (in 100 base pair increments) 600 and 100 base pairs (from top to bottom). The band visualized between molecular markers of 300 and 400 base pairs is the expected 353 base pair human actin PCR product. 


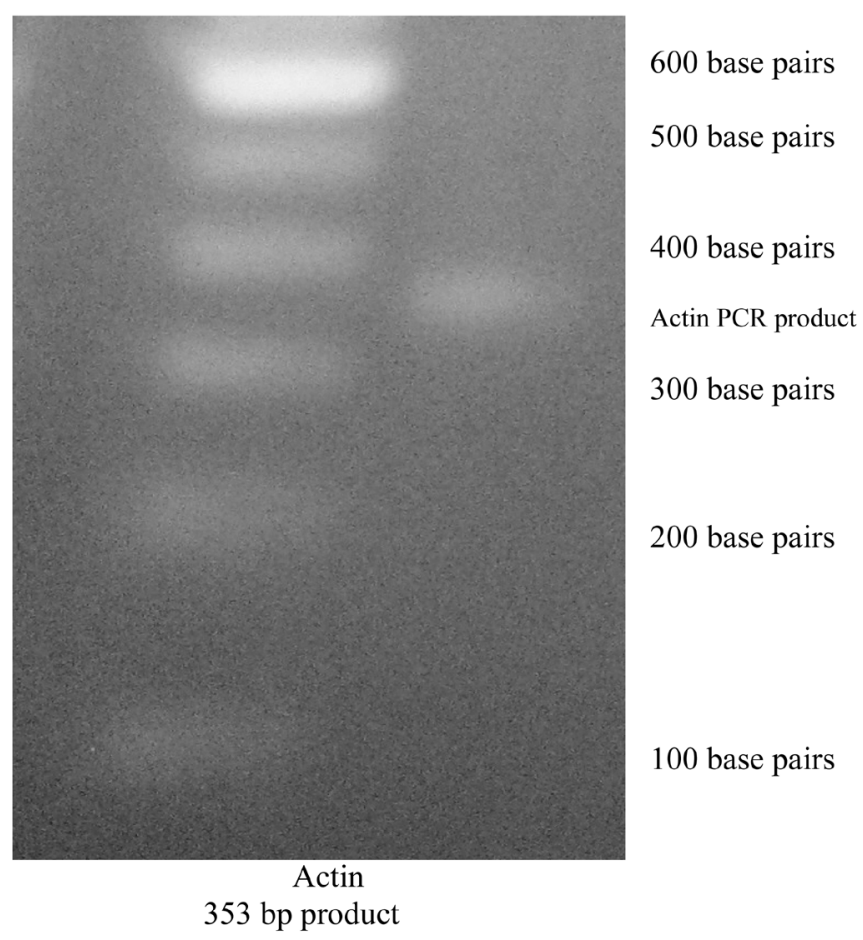

Figure 1. Human actin control in an agarose gel.

LNCaP cells incubated for 24 hours in the presence of $6.25 \mu \mathrm{M}$ of oligos suppressed Bcl-2 expression, and support the finding of comparable biologic activity in both mono- and bispecific oligos measured in the in vitro cell growth inhibition experiments (8). When photographs of the identified product bands were scanned on agarose gels and quantitated using Mipav software, in a series of runs, the greatest expression of bcl-2 was always found in untreated LNCaP cells. Those treated with oligos, whether mono- or bispecific, produced bands which indicated obvious (to the naked eye) suppression. For each oligo evaluated, the greatest amount of suppression measured approached $100 \%$ for the mono-specific $\mathrm{MR}_{4}$; and for the bispecifics $\mathrm{MR}_{24}$ and $\mathrm{MR}_{42}, 86 \%$ and $100 \%$, respectively. Suppression was found in both repeat PCR runs with bcl-2 primers, as well as in repetitive agarose gel quantifications. Figure 2 presents a bcl-2 product band in the expected 127 base pair region which in this run was inhibited $23 \%$ by treatment with the mono-specific $\mathrm{MR}_{4}$, and $86 \%$ and $74 \%$, respectively by bispecifics $\mathrm{MR}_{24}$ and $\mathrm{MR}_{42}$, as measured by Mipav software.

\subsection{V-myc Expression}

Comparable amounts of extracted RNA from LNCaP cells treated with either mono- or bispecific oligos directed against bcl-2 (and EGFR in the bispecifics) were then evaluated by RT-PCR using primers directed against v-myc. When background intensity was subtracted, the relative intensity of all bands corresponding to v-myc representing cells treated with $\mathrm{MR}_{4}, \mathrm{MR}_{24}$ and $\mathrm{MR}_{42}$ compared to controls were increased $170.1 \% \pm 105.6 \%(\mathrm{P}$ $=0.0027), 201.4 \% \pm 101.1 \%(\mathrm{P}=0.0006)$ and $18.4 \% \pm 32.3 \%(\mathrm{P}=0.19)$. These results were pooled from both duplicate PCR runs and multiple gels (a total of six gels were evaluated), and indicate significant enhancement of v-myc activity is produced by oligos which target bcl-2 at the 5' position. A representative band is depicted in Figure 3.

\subsection{K-ras Expression}

Comparable amounts of extracted RNA from LNCaP cells treated with either mono- or bispecific oligos directed against bcl-2 (and EGFR in the bispecifics) were then evaluated by RT-PCR using primers directed to v-myc. When background intensity was subtracted, the relative intensity of all bands corresponding to K-ras representing cells treated with $\mathrm{MR}_{4}, \mathrm{MR}_{24}$ and $\mathrm{MR}_{42}$ compared to controls were $2.1 \% \pm 9.5 \%(\mathrm{P}=0.677),-12.9 \%$ 
200 base pairs

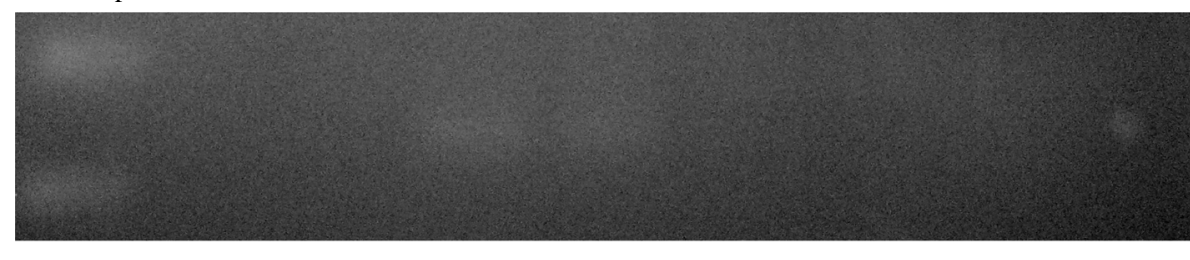

100 base pairs

$\begin{array}{llll}\text { Untreated } & \text { Treated } & \text { Treated } & \text { Treated } \\ \mathrm{MR}_{4} & \mathrm{MR}_{24} & \mathrm{MR}_{42}\end{array}$

Bc1-2 127 bp product

Figure 2. Bcl-2 expression is suppressed by oligos as indicated in a representative agarose gel.

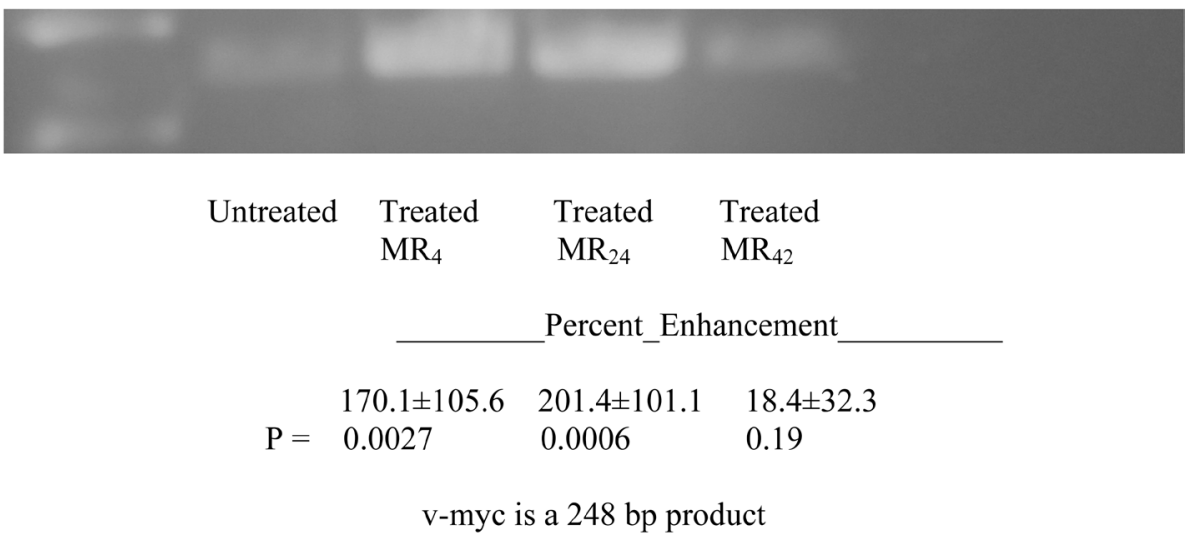

Figure 3. V-myc expression is enhanced by oligos as Indicated in a representative agarose gel.

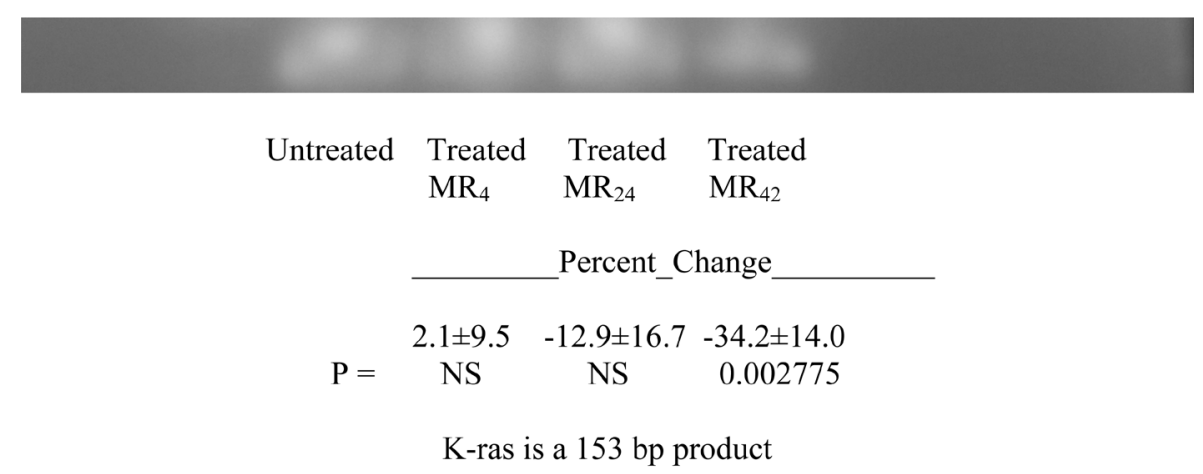

Figure 4. K-ras expression is enhanced by oligos as indicated in a representative agarose gel.

$\pm 16.7 \%(\mathrm{P}=0.174)$ and $-34.2 \% \pm 14.0 \%(\mathrm{P}=0.002775)$. Although treatment with $\mathrm{MR}_{42}$ produced a statistically significant decrease in activity, overall activity was not greatly altered (unlike v-myc). This suggests that effects upon K-ras are not as important as those affecting v-myc, and would imply that should bcl-2 directed therapy be employed, there may be no need for additional regulation of K-ras. A representative band is depicted in Figure 4.

\section{Discussion}

This year (2015) the American Cancer Society (ACS) estimated that in spite of early detection, screening for prostate specific antigen (PSA) and effective treatments for localized disease, in the United States there will be 27,540 deaths from prostate cancer with 220,800 newly diagnosed cases [16]. New types of treatment, including gene therapy and translational inhibition must be developed and employed (probably in combination with tradi- 
tional androgen ablation).

Gene therapy is often promoted as a highly specific and deliverable treatment to control aberrant gene expression by tumor cells (particularly when growth factors, their receptors or apoptosis inhibitors are excessively produced). However, it's now apparent that it's not as specific as previously thought. Antisense oligos consist of nucleotide bases synthesized complimentary in sequence to mRNA. When hybridized to mRNA, they produce a translational arrest of the targeted gene's mRNA expression. Now in clinical trials against a variety of solid tumors, this method is an effective, relatively non-toxic and inexpensive form of therapy and various types of antisense RNAs have been constructed for this purpose. These include the phosphorothioated oligos used in these evaluations and other formulation including 2'-MOE-RNA, morpholinos, siRNA, miRNA etc. Modifications to the oligo backbone and base structure are sometimes used to prevent nuclease degradation, increase half-life or enhance systemic delivery. Some of these derivatives have been evaluated clinically, but all are directed against single gene transcriptional (mRNA) products. In contrast, the oligos discussed in this paper included both monoand bispecific forms, each having a base sequence complementary to and directed against mRNA encoding the apoptosis inhibitor bcl-2, (bispecifics included an additional site directed against epidermal growth factor receptor [EGFR]). Bcl-2 was chosen because it is an inhibitor of apoptosis, therefore in attempts to improve radio [2] [3] and chemotherapy [4] through either restoration or enhancement of this process, oligos specifically directed to suppress it have entered clinical practice. We evaluate bispecific oligos because it would be naïve to believe targeting a single mRNA would be sufficient to produce a clinical response in most tumors, and activity at one site does not affect binding at a second [17], therefore administration of a single oligo having two mRNA targets could have an additional suppressive benefit. Furthermore we have shown that both types of oligos have comparable activity suppressing bcl-2 [17], and we have suggested that bispecifics could even more effective when directed not only against bcl-2, but also towards those genes which are enhanced following bcl-2 suppression, such as KI-67 or as suggested in this study the v-myc oncogene.

Employing oligos to silence or translationally suppress overly expressed genes is apparently more difficult than expected and some unexpected results have been found. While it's understandable that genes which share sequence homology would also be susceptible to antisense oligos, when directed at common sequences, what is not expected are the effects on non-targeted genes, many of which control additional growth regulating pathways. We have also shown that certain complementary stretches of base sequences within the oligo could also produce unanticipated effects on the expression of cell surface antigens (and differentiation proteins). In an early evaluation of bispecifics we reported the enhanced expression of prostate specific membrane antigen (PSMA) [18] when oligos were directed against bcl-2. The unique capacity to produce such changes by these bispecfics (and not a similarly directed monospecific) is attributable to an unusual double strand conformation present in bispecifics and interferon induction (an enhancer of surface antigen expression) [19]. Such expression could enable better recognition and targeting by cytotoxic T cells [19].

Tumors are a mass of genetically unstable heterogenous cells capable of both rapid mutation and selection. Just as bacterial and viral agents develop resistance to chemotherapeutics, tumors cells have similar a capability. In prostate cancer it's thought that hundreds of genes (including those listed here) drive tumor cells to grow, in addition to the effects of androgen acting through the AR (as a transcriptional factor), AR co-activating proteins (p300, IL4, IL6), regulators of apoptosis (bcl-2, clusterin, AKT-1), additional transcriptional factors (MED12) and various autocrine loops (involving transforming growth factor-alpha [TGF- $\alpha$ ], its EGFR binding site, insulin like growth factor [IGF1] and its receptor [IGF-1R]).

Initial evaluation of protein expression associated with compensation regulating the traditional mode of apoptosis (mitochondrial mediated) focused on the bcl-2, bax, bad, clusterin etc. proteins. However, more recent work evaluated proteins associated with tumor cell destruction, via apoptosis, mediated by a secondary route for activation, involving direct signal transduction. This is a process of initiating apoptosis through the binding of activating proteins (ligands) to cell surface receptors. When ligands bind to these receptors they activate a destructive cascade of protein interactions which lead to cell death. These receptors are structurally similar to the tumor necrosis factor receptor (CD95) and also regulate the immune system's cytotoxic T cell response. As mediators of the immune system their expression can have unanticipated effects on certain types of therapy since these proteins, particularly PD-1 and PD-L1, are now being targeted by monoclonal antibodies to treat cancer patients via immune checkpoint blockade, particularly those with melanoma, mesothelioma and lung cancer [20]. Our studies found that PD-1 its ligand PD-L1 and fas-ligand, were all significantly enhanced following bcl-2 suppression and therefore contribute to an additional pathway for compensatory based resistance and control of 
apoptosis [5].

Oligos (produced by Oncogenex Pharmaceuticals) have reached clinical trials for the treatment of prostate cancer (OGX-011), while others remain in preclinical development (OGX-225). Often administered in combination with traditional chemotherapy, these oligos target bcl-2, clusterin (OGX-011 in Phase II testing), heat shock protein 27 (OGX-427) or insulin growth factor binding proteins (OGX-225) [21]. Genta conducted a phase 3 test using oligos (Genasense; oblimersen) directed against bcl-2 for treating melanoma, chronic lymphocytic leukemia and various solid tumors [22], but compensatory effects produced by this agent were not reported. Many represent efforts to restore tumor apoptosis by eliminating suppressive bcl-2 [2]-[4] associated with treatment resistance. Similar approaches are directed at clusterin, but compensatory mechanisms activated by these oligos have not been evaluated. Since derivatives of antisense oligos (siRNA, miRNA) continue to be developed and tested, and while some directed against bcl-2 are in clinical trials, it is important to identify compensatory changes that result.

\section{Conclusion}

Following suppression of bcl-2 by antisense oligos enhanced expression of oncogenes, particularly v-myc provides another mechanism for compensation and increases tumor aggressiveness. This can be related to the finding by other investigators who report the role of v-myc in prostate cancer being related to either its linkage to abnormalities associated with 8p24 [23] or gene amplification [24].

\section{Acknowledgements}

The Cellular Biology laboratory at the Hektoen Institute is supported, in part, by the Blum Kovler Foundation, the Cancer Federation, Safeway/Dominicks Campaign for Breast Cancer Awareness, Lawn Manor Beth Jacob Hebrew Congregation, the Max Goldenberg Foundation, the Pritzker Traubert Family Foundation, the Sternfeld Family Foundation, and the Herbert C. Wenske Foundation.

\section{References}

[1] Rubenstein, M., Hollowell, C.M.P. and Guinan, P. (2011) Inhibition of Bcl-2 by Antisense Oligonucleotides Is Followed by a Compensatory Suppression of Caspase-3 in LNCaP Cells. European Journal of Clinical Medical Oncology, 3, 1-6.

[2] Mu, Z., Hachem, P. and Pollack, A. (2005) Antisense Bcl-2 Sensitizes Prostate Cancer Cells to Radiation. The Prostate, 65, 331-340. http://dx.doi.org/10.1002/pros.20303

[3] Yip, K.W., Mocanu, J.D., Au, P.Y., Sleep, G.T., Busson, D., Yeh, P., Gilbert, W.C., O’Sullivan, R., Gullane, B., Bastianutto, C. and Liu, F.F. (2005) Combination Bcl-2 Antisense and Radiation Therapy for Nasopharyngeal Cancer. Clinical Cancer Research, 11, 8131-8144. http://dx.doi.org/10.1158/1078-0432.CCR-05-1266

[4] Yamanaka, K., Miyake, H., Zangemeister-wittke, U., Jansen, B. and Gleave, M. (2004) Novel Bispecific Antisense Oligonucleotides Inhibiting both Bcl-2 and Bcl-xL Expression Induce Apoptosis and Enhance Chemosensitivity in Human Androgen-Independent Prostate Cancer Cells. Proceedings AACR, 45, Abstract \#2930. (Online)

[5] Rubenstein, M., Hollowell, C.M.P. and Guinan, P. (2015) Following Inhibition of BCL-2 by Antisense Oligonucleotides Compensatory Suppression of Apoptosis Involves the Direct Signal Transduction Pathway of LNCaP Cells. Online Journal of Apoptosis, 4, 1-10. http://dx.doi.org/10.4236/ojapo.2015.41001

[6] Rubenstein, M., Hollowell, C.M.P. and Guinan, P. (2014) Additional Compensatory Mechanisms Altering Antisense Oligonucleotide Suppression of Bcl-2: Effects upon AKT-1 and STAT-3. In Vivo, 28, 867-870.

[7] Rubenstein, M., Hollowell, C.M.P. and Guinan, P. (2011) In LNCaP Cells Enhanced Expression of the Androgen Receptor Compensates for Bcl-2 Suppression by Antisense Oligonucleotides. Therapeutic Advances in Urology, 3, 51-57. http://dx.doi.org/10.1177/1756287211400493

[8] Rubenstein, M., Hollowell, C.M.P. and Guinan, P. (2011) In LNCaP Cells Enhanced Expression of both Androgen Receptor and Co-Stimulatory Protein p300 Compensate for Antisense Oligonucleotide Suppression of Bcl-2. Therapeutic Advances in Urology, 3, 243-250 http://dx.doi.org/10.1177/1756287211426155

[9] Rubenstein, M., Hollowell, C.M.P. and Guinan, P. (2013) Increased Expression of the Androgen Receptor with p300 and IL-6 Coactivators Compensate for Oligonucleotide Suppression of Bcl-2. No Increased CREBBP or IL-4 Expression. Therapeutic Advances in Urology, 5, 85-93. http://dx.doi.org/10.1177/1756287212466281

[10] Rubenstein M., Hollowell, C.M.P. and Guinan, P. (2013) Oligonucleotide Suppression of Bcl-2 in LNCaP Cells Is 
Compensated by Increased Androgen Sensitivity, p53 and Oncogene Activity, and Suppressed Caspase-3. Medical Oncology, 30, 599. http://dx.doi.org/10.1007/s12032-013-0599-3

[11] Rubenstein, M., Hollowell, C.M.P. and Guinan, P. (2014) In LNCaP Inhibition of Bcl-2 by Oligonucleotides Results in Compensatory Changes Involving Apoptosis, Transcription and Immunoregulation. Enliven: Journal of Genetic, Molecular and Cellular Biology, 1, 001.

[12] Sharma, P. and Allison, J. (2015) The Future of Immune Checkpoint Therapy. Science, 348, 56-61. http://dx.doi.org/10.1126/science.aaa8172

[13] www.ncbi.nlm.nih.gov/gene/4609

[14] www.ncbi.nlm.nih.gov/gene/3845

[15] Rubenstein, M., Anderson, K.M., Tsui, P. and Guinan, P. (2006) Synthesis of Branched Antisense Oligonucleotides Having Multiple Specificities: Treatment of Hormone Insensitive Prostate Cancer. Medical Hypotheses, 67, 1375-1380. http://dx.doi.org/10.1016/j.mehy.2006.05.055

[16] Siegal, R.L., Miller, K.D. and Jemal, A. (2015) Cancer Statistics, 2015. CA: A Cancer Journal for Clinicians, 65, 5-29.

[17] Rubenstein, M. and Guinan, P. (2010) Bispecific Antisense Oligonucleotides Have Activity Comparable to Monospecifics in Inhibiting Expression of Bcl-2 in LNCaP Cells. In Vivo, 24, 489-493.

[18] Rubenstein, M., Hollowell, C.M.P. and Guinan, P. (2010) Increased Prostate Specific Membrane Antigen Expression in LNCaP Cells Following Treatment with Bispecific Antisense Oligonucleotides Directed against Bcl-2 and EGFR. Medical Oncology, 27, 1212-1218. http://dx.doi.org/10.1007/s12032-009-9361-2

[19] Rubenstein, M., Hollowell, C.M.P. and Guinan, P. (2011) Bispecific Oligonucleotides May Induce Interferon in LNCaP Cells Enhancing Surface Antigen Expression: Effect of Intra Strand Base Pair Complementarity. In Vivo, 25, 61-67.

[20] http://www.washingtonpost.com/business/studies-merck-drug-keytruda-effective-against-3-cancers/2015/04/19/01d94c fc-e6b4-11e4-8581-633c536add4b_story.html

[21] Oncogenex Website. www.Oncogenex.ca

[22] Kling, J. (2010) Safety Signal Dampens Reception for Mipomersen Antisense. Nature Biotechnology, 28, $295-297$. http://dx.doi.org/10.1038/nbt0410-295

[23] Koh, C.M., Bieberich, C.J., Dang, C.V., Nelson, W.G., Yegnasubramanian, S. and De Marzo, A.M. (2010) MYC and Prostate Cancer. Genes \& Cancer, 1, 617-628. http://dx.doi.org/10.1177/1947601910379132

[24] Ellwood-Yen, K., Gradber, T.G., Wongvipat, J., Iruela-Arispe, M.L., Zhang, J., Matusik, R., Thomas, G.V. and Sawyers, C.L. (2005) Myc-Driven Murine Prostate Cancer Shares Molecular Features with Human Prostate Tumors. Cancer Cell, 4, 223-238. http://dx.doi.org/10.1016/S1535-6108(03)00197-1 